\title{
Weighted Analogue of Inverse Gamma Distribution: Statistical Properties, Estimation and Simulation Study
}

\author{
Afaq Ahmad \\ Department of Mathematical Sciences \\ Islamic University of Science and Technology, Awantipoora, Kashmir \\ baderaafaq@gmail.com \\ S.P Ahmad \\ Department of Statistics \\ University of Kashmir, Srinagar \\ sprvz@yahoo.com
}

\begin{abstract}
In this article we propose a new weighted version of inverse Gamma distribution known as Weighted Inverse Gamma distribution (WIGD). We examine the Length biased and Area biased versions of Weighted Inverse Gamma distribution. Basic structural properties viz moments, mode, moment generating function (mgf), characteristic function (cf), hazard rate function and measures of uncertainty. The parameters of this model are estimated from both classical (namely, maximum likelihood estimator and method of moments, and compare them by using extensive numerical simulations) and Bayesian point of view. The Bayes estimates are estimated by using non-informative Jeffrey's prior and informative Inverse Chi square prior under different types of loss function (symmetric and asymmetric loss functions). Finally, a simulation study has been conducted for comparing weighted inverse gamma distribution with other competing distributions.
\end{abstract}

Keywords: Inverse gamma distribution; Moments; Entropy; Hazard rate; Maximum likelihood estimator; Moment estimator.

\section{Introduction}

The inverse gamma distribution $I G(\alpha, \beta)$ with parameters $\alpha$ and $\beta$, is mentioned infrequently in statistical literature, and usually for a specific purpose. One primary use of the $I G$ distribution is for Bayesian estimation of the mean of the one parameter exponential distribution (see for example Johnson et al. (1995), as well as estimating variance in a normal regression. A number of brief descriptions of the properties of the distribution are available, mostly in text books on Bayesian methods, often in the econometrics literature, e.g., Koch (2007) and Poirier (1995). Kleiber and Kotz (2003) list some basic structural properties of the $I G$ distribution and also model incomes with the distribution. Milevsky and Posner (1998) studied the inverse gamma distribution and point out estimation by method of moments.

The learning of weighted distributions can be used for better comprehension of standard distributions, and provides techniques of spreading distributions for further flexibility to fit the data superior. Rao (1965) proposed the concept of weighted distribution, Patil and Rao (1978) discussed how, for example, truncated distributions and damaged observations can give rise to weighted distributions. Weighted distributions occur frequently in research related to bio-medicine, reliability, ecosystem and branching process can be seen in Patil and Rao (1986), Sharma et al. (2017) studied on Length and Area biased Maxwell distribution, Ahmad et al. (2016) studied length biased Weighted 
Lomax distribution with its applications, Das and Roy (2011) discussed the length-biased Weighted Generalized Rayleigh distribution with its properties, also they develop the length-biased Weighted Weibull distribution.

Suppose $X$ is a non-negative random variable with probability density function (pdf) $f(x)$, and then the pdf of the weighted random variable $X_{w}$ is given by

$$
f_{w}(x)=\frac{w(x) f(x)}{E(w(x))}, x>0
$$

where $w(x)$ be a non-negative weight function. On the support of $X$, where $w(x)>0$ and $\omega$ $=w(x) f(x) d x$ is a normalizing constant that forces $f^{w}(x)$ to integrate to 1 .

Subject upon the choice of weight function $w(x)$, we will get dissimilar weighted distributions. In this paper, we ruminate $w(x)=x^{c}$ and the model is thus achieved is stated as size biased distribution. Evidently when $c=1$, the weight function depends on the length of units of interest, then the resultant distribution is called length biased distribution. Correspondingly, for $c=2$, the resultant distribution is called area biased distribution.

This paper is divided in to two parts: first is to study the structural properties of the weighted inverse gamma distribution (WIG) along with its special cases (for $c=1$ and 2), and second is to estimate the parameters of the model from both classical and Bayesian view point. Finally, simulation study, summary is provided.

\section{Weighted Inverse Gamma distribution}

In this section, we build the pdf of weighted Inverse Gamma distribution by taking the weight function as $w(x)=x^{c}$ and study the behavior of its pdf and hazard function. The probability density function (pdf) and cumulative distribution function (cdf) of the Inverse Gamma distribution is given by

$$
\begin{aligned}
& f(x ; \alpha, \beta)=\frac{\alpha^{\beta}}{\Gamma(\beta)} \frac{1}{x^{\beta+1}} e^{-\frac{\alpha}{x}} \quad, x>0, \alpha>0, \beta>0 \\
& F_{X}(x)=\frac{\gamma\left(\beta, \frac{\alpha}{x}\right)}{\Gamma(\beta)}
\end{aligned}
$$

where $\gamma\left(\beta, \frac{\alpha}{x}\right)$ denotes the upper incomplete gamma function.

Weighted Inverse Gamma distribution (WIGD) is obtained by applying the weights $x^{c}$, to the Classical Inverse Gamma distribution. To define Weighted Inverse Gamma distribution if $X \sim W I G(c, \alpha, \beta)$, then pdf of $X$ is given by

$$
f_{W}(x ; c, \alpha, \beta)=\frac{\alpha^{\beta-c}}{\Gamma(\beta-c)} \frac{1}{x^{\beta-c+1}} e^{-\frac{\alpha}{x}}, x>0, \alpha>0, \beta>c, c>0
$$

By substituting $c=1$ and $c=2$ in (4), we get the pdfs of length biased Inverse Gamma (LBIG) and area biased Inverse Gamma (ABIG) distributions respectively. Figure (1.1) 
represents shapes of Weighted Inverse gamma distribution for different values of parameters.

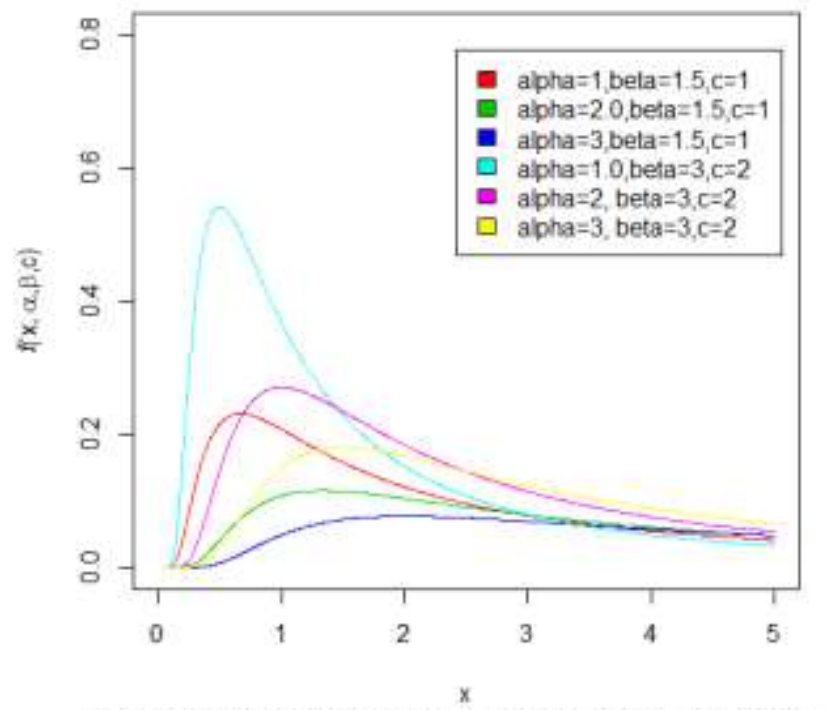

Figure 1.1: Pdf of WIG Distribution under different values to parameters

The cdf, reliability function and hazard function corresponding to the pdf (4) are respectively given by

$$
\begin{gathered}
F_{W X}(x)=\frac{\gamma\left(\beta-c, \frac{\alpha}{x}\right)}{\Gamma(\beta-c)} \\
R(x)=1-\frac{\gamma\left(\beta-c, \frac{\alpha}{x}\right)}{\Gamma(\beta-c)} \\
h(x)=\frac{\alpha^{\beta-c}}{\left(\Gamma(\beta-c)-\gamma\left(\beta-c, \frac{\alpha}{x}\right)\right)} \frac{1}{x^{\beta-c+1}} e^{-\frac{\alpha}{x}}
\end{gathered}
$$

where $\Gamma a=\int_{0}^{\infty} e^{-x} x^{a-1} d x$ is gamma function and $\gamma(a, b)=\int_{0}^{b} e^{-x} x^{a-1} d x$ is the upper incomplete gamma function.

\section{Relationship with other distributions}

Some well-known theoretical distributions can be derived from the proposed WIG distribution. For instance;

1. For $c=0$, equation (4) reduces to the inverse gamma distribution.

2. For $\beta=1$ and $c=0$, equation (4) reduces to the inverse Exponential distribution.

3. For $\beta=\frac{\beta}{2}, \alpha=\frac{1}{2}$ and $c=0$, equation (4) reduces to Inverse Chi-Square distribution. 
4. For $\beta=\frac{1}{2}, \alpha=\frac{l}{2}$ and $c=0$, equation (4) reduces to Levy distribution.

\subsection{Mode of Weighted Inverse Gamma distribution}

The pdf of $\operatorname{WIG}(c, \alpha, \beta)$ is unimodal for given $c, \alpha$ and $\beta$ and achieve its maximum at $X_{\text {mode }}=\frac{\alpha}{\beta-c+1}$.

\section{Proof:}

For the pdf (4),

$$
f_{w}^{\prime}(x)=\frac{\partial}{\partial x} f_{w}(x)=\left(\frac{c-\beta-1}{x}+\frac{\alpha}{x^{2}}\right) f_{w}(x)
$$

The mode of the weighted inverse gamma can be readily obtained from

$$
f_{w}^{\prime}(x)=0 \quad \Rightarrow\left(\frac{c-\beta-1}{x}+\frac{\alpha}{x^{2}}\right) f_{w}(x)=0
$$

Since $f_{w}(x) \neq 0, \forall x>0$, we get

$$
\left(\frac{c-\beta-1}{x}+\frac{\alpha}{x^{2}}\right)=0
$$

Thus the pdf (4) achieve its maximum at $X_{\text {mode }}=\frac{\alpha}{\beta-c+1}$.

\subsection{Moments of Weighted Inverse Gamma distribution}

Moments helps to determine many properties of the distribution such as Averages, dispersion, skewness and kurtosis. The $\mathrm{r}^{\text {th }}$ moment about origin of the Weighted Inverse Gamma distribution is given by

$$
\mu_{r}^{\prime}=\frac{\alpha^{\beta-c}}{\Gamma(\beta-c)} \frac{\Gamma(\beta-c-r)}{\alpha^{\beta-c-r}}, r=1,2,3, \ldots
$$

The mean and variance of Weighted Inverse Gamma distribution is given by

$$
\mu=\frac{\alpha}{\beta-c-1} \sigma^{2}=\frac{\alpha^{2}}{(\beta-c-1)^{2}(\beta-c-2)}
$$

By substituting $\mathrm{c}=1,2$ in (5) the mean and variance of Length biased Inverse Gamma and Area biased inverse gamma distribution are $\left(\mu=\frac{\alpha}{\beta-2}, \sigma^{2}=\frac{\alpha^{2}}{(\beta-2)^{2}(\beta-3)}\right)$ and $\left(\mu=\frac{\alpha}{\beta-3}, \sigma^{2}=\frac{\alpha^{2}}{(\beta-3)^{2}(\beta-4)}\right)$. The coefficient of skewness and kurtosis of $W I G(c, \alpha, \beta)$ are given by 


$$
\begin{aligned}
& S_{k}=\beta_{1}=\frac{4 \sqrt{\beta-c-2}}{\beta-c-3} \\
& K_{r}=\beta_{2}=\frac{30 \beta-30 c-66}{(\beta-c-3)(\beta-c-4)}
\end{aligned}
$$

By putting $c=1,2$ in (6) and (7), the skewness and kurtosis of LBIG and ABIG distribution are

$$
\left(\beta_{1}=\frac{4 \sqrt{\beta-3}}{\beta-4}, \beta_{2}=\frac{30 \beta-96}{(\beta-4)(\beta-5)}\right)
$$

and

$$
\left(\beta_{1}=\frac{4 \sqrt{\beta-4}}{\beta-5}, \beta_{2}=\frac{30 \beta-126}{(\beta-5)(\beta-6)}\right) \text { respectively. }
$$

\section{Corollary 2.1.}

The Moment generating function of $\operatorname{WIG}(c, \alpha, \beta)$ does not exists.

\section{Corollary 2.2.}

The characteristic function of $\operatorname{WIG}(c, \alpha, \beta)$ is given by

$$
\phi_{X}(t)=\frac{2(i \alpha t)^{\frac{\beta-c}{2}}}{\Gamma(\beta-c)} K_{\beta}(\sqrt{-4 i \alpha t})
$$

\section{Proof:}

By definition of moment generating function

$$
\phi_{X}(t)=E\left(e^{i t x}\right)=\int_{0}^{\infty} e^{i t x} f_{w}(x) d x
$$

Substituting the pdf of $W I G(c, \alpha, \beta)$ in above expression we get the required result.

$$
\begin{gathered}
\phi_{X}(t)=E\left(e^{i t x}\right)=\frac{\alpha^{\beta-c}}{\Gamma(\beta-c)} \int_{0}^{\infty} e^{i t x} \frac{1}{x^{\beta-c+1}} e^{-\frac{\alpha}{x}} d x \\
\phi_{X}(t)=\frac{2(i \alpha t)^{\frac{\beta-c}{2}}}{\Gamma(\beta-c)} K_{\beta}(\sqrt{-4 i \alpha t})
\end{gathered}
$$

where

$$
\begin{aligned}
K_{\beta}(\sqrt{z})= & \frac{1}{2}\left(\frac{1}{2} z\right)^{-\beta} \sum_{k=0}^{\beta-1} \frac{(\beta-k-1) !}{k !}\left(-\frac{1}{4} z^{2}\right)^{k}+(-1)^{\beta+1} \ln \left(\frac{1}{2 z}\right) I_{\beta}(z)+ \\
& (-1)^{\beta}\left(\frac{1}{2}\right)\left(\frac{1}{2} z\right)^{\beta} \sum_{k=0}^{\infty}\left[\psi(k+1)+\psi(\beta+k+1] \frac{\left(\frac{1}{2} z^{2}\right)^{k}}{k !(\beta+k) !}\right.
\end{aligned}
$$

is modified Bessel function of second kind and $\psi($.$) is digamma function.$

\subsection{Measures of Uncertainty}

The entropy of a random variable $\mathrm{X}$ with probability density $W I G(c, \alpha, \beta)$ is a measure of variation of the uncertainty. 


\subsubsection{Shannon's Entropy}

The Shannon's entropy is given by

$$
H(X)=-E\left(\log \left(f_{w}(x)\right)\right.
$$

Using pdf (4) in above equation we get

$$
\begin{aligned}
& H(X)=\log \Gamma(\beta-c)-(\beta-c) \log \alpha-(\beta-c+1) E(\log (1 / x))+\alpha E(1 / x) \\
& =(\beta-c)+\log (\alpha \Gamma(\beta-c))-(\beta-c+1) \psi(\beta-c)
\end{aligned}
$$

where

$$
\psi(x)=\frac{\Gamma^{\prime} x}{\Gamma x} \text { and } \int_{0}^{\infty} \log (x) x^{p-1} e^{-x} d x=\Gamma^{\prime} p
$$

\subsubsection{Rényi entropy}

The Rényi entropy (1960), denoted by $I_{R}(\rho)$ is defined as

$$
I_{R}(\rho)=\frac{1}{1-\rho} \log \left\{\int_{-\infty}^{\infty} f_{w}^{\rho}(x) d x\right\}
$$

where $\rho>0$ and $\rho \neq 1$.

By substituting pdf (4) in above expression, we obtain

$$
\begin{aligned}
& I_{R}(\rho)=\frac{1}{1-\rho} \log \left\{\int_{0}^{\infty}\left(\frac{\alpha^{\beta-c}}{\Gamma(\beta-c)}\right)^{\rho} \frac{1}{x^{\rho(\beta-c+1)}} e^{-\frac{\rho \alpha}{x}} d x\right\} \\
& =\frac{1}{1-\rho} \log \left\{\frac{\alpha^{\rho(\beta-c)}}{\Gamma^{\rho}(\beta-c)} I(\rho(\beta-c+1)-1, \rho \alpha)\right\}
\end{aligned}
$$

Where

$$
I(a, b)=\int_{0}^{\infty} \frac{1}{x^{a+1}} e^{-\frac{b}{x}} d x=\frac{\Gamma a}{b^{a}}
$$

\section{Methods of Estimation}

In this section, parameters of weighted inverse gamma distribution are estimated by various methods of estimation viz, method of moments, maximum likelihood estimation and Bayesian method of estimation.

\subsection{Method of Moments}

In order to estimate the unknown parameters of $\operatorname{WIG}(c, \alpha, \beta)$ model by the method of moments, we equate the sample moments with the corresponding population moments.

$$
\mu_{r}^{\prime}=\frac{1}{n} \sum_{i=0}^{n} x_{i}^{r}
$$

Replacing sample moments with population moments, we get 


$$
\begin{gathered}
\frac{1}{n} \sum_{i=1}^{n} x_{i}=\mu_{1}^{\prime} \\
\Rightarrow \bar{x}=\frac{\alpha}{\beta-c-1} \\
\text { and } \frac{1}{n} \sum_{i=1}^{n} x_{i}^{2}=\mu_{2}^{\prime} \\
\Rightarrow \frac{1}{n} \sum_{i=1}^{n} x_{i}^{2}-\bar{x}^{2}=\mu_{2} \\
\Rightarrow s^{2}=\frac{\alpha^{2}}{(\beta-c-1)^{2}(\beta-c-2)}
\end{gathered}
$$

On solution the equations (10) and (11) we obtain the estimates for $\alpha$ and $\beta$ say $\hat{\alpha}$ and $\hat{\beta}$ respectively.

\subsection{Maximum Likelihood Estimation}

Let $x_{1}, x_{2}, x_{3} \ldots \ldots x_{n}$ be a random sample from the Weighted Inverse Gamma distribution, then the corresponding likelihood function is given as

$$
l(X ; \alpha, \beta)=\left(\frac{\alpha^{\beta-c}}{\Gamma(\beta-c)}\right)^{n} \prod_{i=1}^{n}\left(\frac{1}{x_{i}^{\beta-c+1}}\right) \exp \left(-\frac{\alpha}{\sum_{i=1}^{n} x_{i}}\right)
$$

The log-likelihood function is given as:

$$
\log l(x ; \alpha, \beta)=n(\beta-c) \log \alpha-n \log \Gamma(\beta-c)+\sum_{i=1}^{n} \log \left(\frac{1}{x_{i}^{\beta-c+1}}\right)-\frac{\alpha}{\sum_{i=1}^{n} x_{i}}
$$

Now, differentiate above equation with respect to parameters $\alpha$ and $\beta$ and equate to zero, we obtain the normal equations

$$
\begin{aligned}
& \frac{\partial \log l}{\partial \alpha}=0 \Rightarrow \frac{n(\beta-c)}{\alpha}-\frac{1}{\sum_{i=1}^{n} x_{i}}=0 \\
& \frac{\partial \log l}{\partial \beta}=0 \Rightarrow n \log \alpha-n\left(\log (\beta-c)-\frac{1}{2(\beta-c)}\right)+\sum_{i=1}^{n} \log x_{i}=0
\end{aligned}
$$

The MLE $\hat{\eta}=(\hat{\alpha}, \hat{\beta})$ of $\eta=(\alpha, \beta)$ is obtained by solving the above nonlinear system of equations. It is usually more convenient to use nonlinear optimization algorithms such as quasi-Newton algorithm to numerically maximize the log likelihood function given in (12). Applying the usual large sample approximation, the MLE $\hat{\eta}$ can be treated as being 
approximately bivariate normal with variance-covariance matrix equal to the inverse of the expected information matrix, i.e.

$$
\sqrt{n}(\hat{\eta}-\eta) \rightarrow N\left(0, n I^{-1}(\eta)\right)
$$

where $I^{-1}(\eta)$ is the limiting variance-covariance matrix of $\hat{\eta}$.

\subsection{Bayesian Method of Estimation}

In this section, we construct Bayes estimator of the scale parameter $\alpha$ of WIG distribution using non informative Jeffrey's prior and informative Inverse Chi square prior under different loss functions.

\subsubsection{Posterior distribution using Jeffrey's prior}

Assuming that $\alpha$ has Jeffery prior i.e $g(\alpha) \propto \frac{1}{\alpha}$ the posterior distribution is given by

$$
P_{J}(\alpha \mid \underset{-}{x})=\frac{t_{1}^{n(\beta-c)}}{\Gamma(n(\beta-c))} \alpha^{n(\beta-c)-1} e^{-\alpha t_{1}}
$$

which is density function of gamma distribution with shape parameter $\left(t_{1}\right)$ and scale parameter $n(\beta-c)$, where $t_{1}=\frac{1}{\sum_{i=1}^{n} x_{i}}$.

The Bayes estimate of $\alpha$ using Jeffrey's prior under SELF, Entropy and LINEX are given by

$$
\begin{gathered}
\hat{\alpha}_{S}=\frac{n(\beta-c)}{t_{1}} \\
\hat{\alpha}_{E}=\frac{(n(\beta-c)-1)}{t_{1}}
\end{gathered}
$$

and

$$
\hat{\alpha}_{L}=\frac{1}{a} \log \left(\frac{a+t_{1}}{t_{1}}\right)^{n(\beta-c)}
$$

\subsubsection{Posterior Risk Functions:}

The risk functions of the estimators $\hat{\alpha}_{S}, \hat{\alpha}_{E}$ and $\hat{\alpha}_{L}$ relative to SELF, entropy loss function and LINEX loss function are denoted by $R\left(\hat{\alpha}_{S}\right), R\left(\hat{\alpha}_{E}\right)$ and $R\left(\hat{\alpha}_{L}\right)$ are given by

$$
\begin{gathered}
R\left(\hat{\alpha}_{S}\right)=\hat{\alpha}^{2}+\frac{(n(\beta-c)+1)(n(\beta-c))}{t_{1}^{2}}-\frac{2 n(\beta-c) \hat{\alpha}}{t_{1}} \\
R\left(\hat{\alpha}_{E}\right)=\frac{\hat{\alpha} t_{1}}{(n(\beta-c))}-\log \hat{\alpha}+\psi(n(\beta-c))-1
\end{gathered}
$$




$$
R\left(\hat{\alpha_{E}}\right)=e^{a \hat{\alpha}}\left(\frac{t_{1}}{t_{1}+a}\right)^{n(\beta-c)}-a \hat{\alpha}+\frac{a(n(\beta-c))}{t_{1}}-1
$$

where $\psi(n(\beta-c))$ is a digamma function.

\section{Lemma:}

For given posterior distribution (15), we have

$$
\begin{aligned}
E(\alpha \mid \underset{-}{x}) & =\sum_{i=1}^{n} x_{i}(n(\beta-c)) \\
E\left(\alpha^{2} \mid \underset{-}{x}\right) & =\left(\sum_{i=1}^{n} x_{i}\right)^{2}(n(\beta-c))(n(\beta-c)+1) \\
E\left(\alpha^{-1} \mid \underset{-}{x}\right) & =\frac{1}{\sum_{i=1}^{n} x_{i}(n(\beta-c)-1)} \\
E\left(\left.\alpha^{-2}\right|_{-} ^{x}\right) & =\frac{1}{\left(\sum_{i=1}^{n} x_{i}\right)^{2}(n(\beta-c)-1)(n(\beta-c)-2)}
\end{aligned}
$$

\subsubsection{Posterior distribution using inverse Chi-square prior}

Assuming that $\alpha$ has inverse chi-square defined by $g(\alpha)=\frac{(v / 2)^{\frac{v}{2}}}{\Gamma(v / 2)} \frac{e^{-\frac{v}{2 x}}}{x^{1+v / 2}}$ the posterior distribution is given by

$$
P_{I C}(\alpha \mid x)=\frac{t_{2}^{n(\beta-c)+2}}{\Gamma(n(\beta-c)+2)} \alpha^{n(\beta-c)+1} e^{-t_{2} \alpha}
$$

which is density function of gamma distribution with shape parameter $\left(t_{2}\right)$ and scale parameter $(n(\beta-c)+2)$, where $t_{2}=\left(\theta+\frac{1}{\sum_{i=1}^{n} x_{i}}\right)$.

The Bayes estimate of $\alpha$ using Inverse Chi-square prior under SELF, Entropy and LINEX are given by

$$
\begin{gathered}
\hat{\alpha}_{S}=\frac{n(\beta-c)+2}{t_{2}} \\
\hat{\alpha}_{E}=\frac{(n(\beta-c)+1}{t_{2}}
\end{gathered}
$$

And

$$
\hat{\alpha}_{L}=\frac{1}{a} \log \left(\frac{a+t_{2}}{t_{2}}\right)^{n(\beta-c)+2}
$$




\subsubsection{Posterior Risk Functions:}

The risk functions of the estimators $\hat{\alpha}_{S}, \hat{\alpha}_{E}$ and $\hat{\alpha}_{L}$ relative to SELF, entropy loss function and LINEX loss function are denoted by $R\left(\hat{\alpha_{S}}\right), R\left(\hat{\alpha_{E}}\right)$ and $R\left(\hat{\alpha_{L}}\right)$ are given by

$$
\begin{gathered}
R\left(\hat{\alpha}_{S}\right)=\hat{\alpha}^{2}+\frac{(n(\beta-c)+3)(n(\beta-c)+2)}{t_{2}{ }^{2}}-\frac{2(n(\beta-c)+2) \hat{\alpha}}{t_{2}} \\
R\left(\hat{\alpha}_{E}\right)=\frac{\hat{\alpha} t_{2}}{(n(\beta-c)+1)}-\log \hat{\alpha}+\psi(n(\beta-c)+2)-1 \\
R\left(\hat{\alpha}_{E}\right)=e^{a \hat{\alpha}}\left(\frac{t_{2}}{a+t_{2}}\right)^{n(\beta-c)+2}-a \hat{\alpha}+\frac{a(n(\beta-c)+2)}{t_{2}}-1
\end{gathered}
$$

where $\psi(n(\beta-c)+2)$ is a digamma function.

\section{Simulation Study}

The most common and simplest method for generating random sample is based on the inverse cumulative distribution function (cdf). For arbitrary cdf, define $G^{-1}(u)=\min \{x$; $G(x) \geq u\}$. The inverse cdf method can't be directly applied for WIG distribution because of the closed form expression for its quantile function is not available. Here, we intend to use Newton's method for the calculation of the quantile function numerically. The algorithm used for this determination is as follows:

\section{Algorithm:}

Step 1. Set $n, c, \alpha, \beta$ and initial value $x^{0}$.

Step 2. Generate $U \sim \operatorname{Uniform}(0,1)$.

Step 3. Update $x^{0}$ by using the Newton's formula, $x^{*}=x^{0}-R\left(x^{0}, c, \alpha, \beta\right)$, where $R\left(x^{0}, c, \alpha, \beta\right)=\frac{G\left(x^{0}, c, \alpha, \beta\right)-U}{g\left(x^{0}, c, \alpha, \beta\right)}$, where G(.) and $\mathrm{g}($.$) are cdf and pdf WIG distribution$ respectively.

Step 4. If $\left|x^{0}-x^{*}\right|<\epsilon$, (very small, $\in>0$ tolerance limit), store $x=x^{*}$ as a sample from $W I G(c, \alpha, \beta)$.

Step 5. If $\left|x^{0}-x^{*}\right|>\in$, then set $x^{0}=x^{*}$ and go to step 3 .

Step 6. Repeat steps $2-5, n$ times for $x_{1}, x_{2}, x_{3}, \ldots, x_{n}$ respectively.

On the basis of generated sample from the above algorithm, we use R code and check the goodness of fit of the LWIG and AWIG distributions and compare the appropriateness with some other class of weighted distributions. 
We apply KS test and AIC criterion for goodness of fit. The summary of the fitting is presented in Table 4.1. From the table, we observe that the family of weighted Inverse Gamma distribution is suitable for modelling than the other competing distributions.

Table 4.1: Estimation of Parameters and Comparison Criteria

\begin{tabular}{|c|c|c|c|c|c|c|c|}
\hline $\begin{array}{c}\text { Distributio } \\
n\end{array}$ & $\begin{array}{c}\text { Paramete } \\
\mathrm{r} \\
\text { estimate }\end{array}$ & $\begin{array}{c}\text { Standar } \\
\mathrm{d} \\
\text { Error }\end{array}$ & $-2 \log l$ & AIC & $\mathrm{BIC}$ & AICC & $\begin{array}{c}\text { Shanno } \\
n \\
\text { Entropy }\end{array}$ \\
\hline IGD & $\begin{array}{c}\hat{\alpha}= \\
1.1643 \\
\hat{\beta} \\
=0.8367\end{array}$ & $\begin{array}{l}0.3823 \\
0.2050\end{array}$ & $\begin{array}{c}136.47 \\
9\end{array}$ & $\begin{array}{c}140.47 \\
9\end{array}$ & $\begin{array}{c}139.27 \\
3\end{array}$ & $\begin{array}{c}141.02 \\
4\end{array}$ & 2.729 \\
\hline LBIGD & $\begin{array}{c}\hat{\alpha}= \\
1.8826 \\
\hat{\beta} \\
=2.3149\end{array}$ & $\begin{array}{l}0.5814 \\
0.3351\end{array}$ & $\begin{array}{c}109.48 \\
8\end{array}$ & $\begin{array}{c}113.48 \\
8\end{array}$ & $\begin{array}{c}112.28 \\
2\end{array}$ & $\begin{array}{c}114.03 \\
3\end{array}$ & 2.1806 \\
\hline ABIGD & $\begin{array}{c}\hat{\alpha}= \\
1.9084 \\
\hat{\beta} \\
=3.4394\end{array}$ & $\begin{array}{l}0.5841 \\
0.3694\end{array}$ & $\begin{array}{c}100.87 \\
1\end{array}$ & $\begin{array}{c}104.87 \\
1\end{array}$ & $\begin{array}{c}103.66 \\
5\end{array}$ & $\begin{array}{c}105.41 \\
6\end{array}$ & 2.017 \\
\hline $\begin{array}{c}\text { Inverse Chi } \\
\text { Square }\end{array}$ & $\begin{array}{c}\hat{\alpha} \\
=0.9387\end{array}$ & 0.1705 & $\begin{array}{c}219.61 \\
0\end{array}$ & $\begin{array}{c}221.61 \\
0\end{array}$ & $\begin{array}{c}220.98 \\
9\end{array}$ & $\begin{array}{c}221.78 \\
3\end{array}$ & 4.392 \\
\hline $\begin{array}{c}\text { Inverse } \\
\text { Exponentia } \\
1\end{array}$ & $\begin{array}{c}\hat{\alpha} \\
=1.3106\end{array}$ & 0.2621 & $\begin{array}{c}123.08 \\
6\end{array}$ & $\begin{array}{c}125.08 \\
6\end{array}$ & $\begin{array}{c}124.48 \\
3\end{array}$ & $\begin{array}{c}125.25 \\
9\end{array}$ & 2.461 \\
\hline Levy & $\begin{array}{c}\hat{\alpha} \\
=0.0357\end{array}$ & 0.0101 & $\begin{array}{c}354.68 \\
1\end{array}$ & $\begin{array}{c}356.68 \\
1\end{array}$ & $\begin{array}{c}356.07 \\
8\end{array}$ & $\begin{array}{c}356.85 \\
4\end{array}$ & 7.093 \\
\hline
\end{tabular}

From Table 4.1, it has been observed that the family of Weighted Inverse Gamma distribution have the lesser AIC, AICC and BIC values as compared to other competing distributions. Hence we can concluded that the Weighted Inverse Gamma distribution leads to a better fit and is suitable for modeling than the other competing distributions.

\section{Application}

The data set represents the survival times (in days) of 72 guinea pigs infected with virulent tubercle bacilli, observed and reported by Bjerkedal (1960). The data are as follows: $0.1,0.33,0.44,0.56,0.59,0.59,0.72,0.74,0.92,0.93,0.96,1,1,1.02,1.05,1.07,1.07$, $1.08,1.08,1.08,1.09,1.12,1.13,1.15,1.16,1.2,1.21,1.22,1.22,1.24,1.3,1.34,1.36$, $1.39,1.44,1.46,1.53,1.59,1.6,1.63,1.68,1.71,1.72,1.76,1.83,1.95,1.96,1.97,2.02$, $2.13,2.15,2.16,2.22,2.3,2.31,2.4,2.45,2.51,2.53,2.54,2.78,2.93,3.27,3.42,3.47$, $3.61,4.02,4.32,4.58,5.55,2.54,0.77$. 


\begin{tabular}{|c|c|c|c|c|c|c|}
\hline Distribution & $\begin{array}{l}\text { Parameter } \\
\text { Estimate }\end{array}$ & $\begin{array}{l}\text { Standard } \\
\text { Error }\end{array}$ & $-2 \log l$ & AIC & BIC & AICC \\
\hline IGD & $\begin{array}{c}\hat{\alpha}= \\
0.2730 \\
\hat{\beta}=1.3008\end{array}$ & $\begin{array}{c}0.1868 \\
1.71105\end{array}$ & 112.7648 & 118.764 & 124.5007 & 119.2865 \\
\hline LBIGD & $\begin{array}{c}\hat{\alpha}= \\
0.0332 \\
\hat{\beta}=0.0421\end{array}$ & $\begin{array}{l}0.0223 \\
0.0166\end{array}$ & 77.04523 & 83.0452 & 88.7812 & 83.5669 \\
\hline ABIGD & $\begin{array}{c}\hat{\alpha}=0.0151 \\
\hat{\beta}= \\
0.0343\end{array}$ & $\begin{array}{l}0.0028 \\
0.0051\end{array}$ & 41.6281 & 47.6281 & 53.3641 & 48.1498 \\
\hline $\begin{array}{c}\text { Inverse Chi } \\
\text { Square }\end{array}$ & $\begin{array}{c}\hat{\alpha} \\
=11.4351\end{array}$ & 6.5347 & 114.6634 & 116.6634 & 118.5760 & 116.7467 \\
\hline $\begin{array}{c}\text { Inverse } \\
\text { Exponential }\end{array}$ & $\begin{array}{c}\hat{\alpha} \\
=13.1806\end{array}$ & 18.4316 & 94.6973 & 98.6973 & 102.5213 & 98.9526 \\
\hline Levy & $\hat{\alpha}=0.1772$ & 0.0289 & 246.1711 & 248.1711 & 250.0837 & 248.2544 \\
\hline
\end{tabular}

From above Table, it has been clearly observed that the family of Weighted Inverse Gamma distribution have the lesser AIC, AICC and BIC values as compared to other competing distributions. Hence we can concluded that the Weighted Inverse Gamma distribution leads to a better fit and is suitable for modeling than the other competing distributions.

\section{References}

1. Ahmad, A., Ahmad, S.P. and Ahmed, A., (2016). "Length Biased Weighted Lomax distribution: Statistical Properties and Applications", Pakistan Journal of Statistics and Operations Research, vol. 12(2), pp. 245-255.

2. Bjerkedal, T. (1960). "Acquisition of resistance in guinea pigs infected with different doses of virulent tubercle bacilli”, American Journal of Epidemiol vol.72 (1), pp. $130-148$.

3. Das, K. K. and Roy, T. D. (2011). “Applicability of Length Biased Weighted Generalized Rayleigh distribution", Advances in Applied Science Research, 2 (4): 320-327.

4. Fisher, R.A., 1934. "The effect of methods of ascertainment upon the estimation of frequencies", Ann. Eng. 6, 13-25.

5. Johnson, N.L., Kotz, S. and Balakrishna, N. (1995). "Continuous Univariate distributions", $2^{\text {nd }}$ ed., vol. 2, New York: Wiley.

6. Kleiber, C and Kotz, S. (2003). "Statistical size distributions in economics and actuarial sciences", New York: Wiley. 
7. Koch, K. (2007). "Introduction to Bayesian statistics", Heidelberg: Springer.

8. Milevsky, M.A and Posner, S.E., (1998). "Asian options, the sum of log-normals and the reciprocal gamma distribution", The Journal of Financial and Quantitative Analysis, vol. 33(2), 203-218.

9. Patil, G.P., Rao, C.R., (1978). Weighted distributions and size-biased sampling with applications to wildlife and human families. Biometrics 34, 179-189.

10. Patil, G.P., Rao, C.R., Ratnaparkhi, M.V., (1986). "On discrete weighted distributions and their use in model choice for observed data", Communication in Statistics - Theory and Methods, vol. 15(3), 907-918.

11. Poirier, D. J. (1995). "Intermediate statistics and econometrics", Cambridge: MIT Press.

12. Rao, C.R., (1965). "On discrete distributions arising out of methods of ascertainment. Classical and Contagious Discrete Distributions”, Eds. G.P. Patil Pergamon Press and Statistical Publishing Society -Calcutta.

13. Renyi, A., (1960): "On Measures of Information and Entropy", Proceedings of the fourth Berkeley Symposium on Mathematics, Statistics and Probability, pp. 547561.

14. Sharma, V.K., Dey, S., Singh, S.K. and Uzma, M., (2017). "On Length and Area biased Maxwell distribution", Communication in Statistics-Simulation and Computation, http://dx.doi.org/10.108/03610918.2017.1317804. 